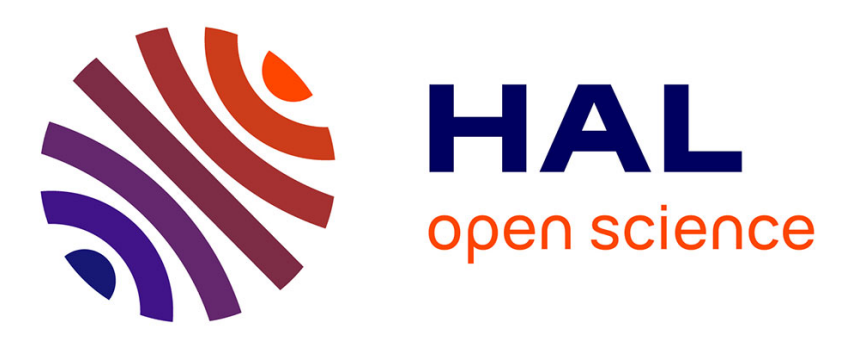

\title{
Derivation of a coupled system of Korteweg-de Vries equations describing ultrashort soliton propagation in quadratic media by using a general Hamiltonian for multilevel atoms
}

\author{
Hervé Leblond, Houria Triki, Dumitru Mihalache
}

\section{To cite this version:}

Hervé Leblond, Houria Triki, Dumitru Mihalache. Derivation of a coupled system of Korteweg-de Vries equations describing ultrashort soliton propagation in quadratic media by using a general Hamiltonian for multilevel atoms. Physical Review A : Atomic, molecular, and optical physics [1990-2015], 2012, 85 (5), pp.053826. 10.1103/PhysRevA.85.053826 . hal-03187673

\section{HAL Id: hal-03187673 \\ https://univ-angers.hal.science/hal-03187673}

Submitted on 1 Apr 2021

HAL is a multi-disciplinary open access archive for the deposit and dissemination of scientific research documents, whether they are published or not. The documents may come from teaching and research institutions in France or abroad, or from public or private research centers.
L'archive ouverte pluridisciplinaire HAL, est destinée au dépôt et à la diffusion de documents scientifiques de niveau recherche, publiés ou non, émanant des établissements d'enseignement et de recherche français ou étrangers, des laboratoires publics ou privés. 


\title{
Derivation of a coupled system of Korteweg-de Vries equations describing ultrashort soliton propagation in quadratic media by using a general Hamiltonian for multilevel atoms
}

\author{
Hervé Leblond, ${ }^{1}$ Houria Triki, ${ }^{2}$ and Dumitru Mihalache ${ }^{1,3,4}$ \\ ${ }^{1}$ LUNAM Université, Université d'Angers, Laboratoire de Photonique d'Angers, EA 4464, 2 Bd. Lavoisier, 49045 Angers Cedex 01, France \\ ${ }^{2}$ Radiation Physics Laboratory, Department of Physics, Faculty of Sciences, Badji Mokhtar University, P. O. Box 12, 23000 Annaba, Algeria \\ ${ }^{3}$ Academy of Romanian Scientists, 54 Splaiul Independentei, 050094 Bucharest, Romania \\ ${ }^{4}$ Horia Hulubei National Institute for Physics and Nuclear Engineering, 30 Reactorului, Magurele-Bucharest, 077125, Romania
}

(Received 29 March 2012; published 22 May 2012)

\begin{abstract}
We consider the propagation of ultrashort solitons in noncentrosymmetric quadratically nonlinear optical media described by a general Hamiltonian of multilevel atoms. We use a long-wave approximation to derive a coupled system of Korteweg-de Vries equations describing ultrashort soliton evolution in such materials. This model was derived by using a rigorous application of the reductive perturbation formalism (multiscale analysis). The study of linear eigenpolarizations in the degenerate case and the corresponding formation of half-cycle solitons from few-cycle-pulse inputs are also presented.
\end{abstract}

DOI: 10.1103/PhysRevA.85.053826

PACS number(s): 42.65.Tg, 42.65.Sf, 47.20.Ky

\section{INTRODUCTION}

Ultrashort pulses with durations of only a few periods of the optical radiation have become intensively used in the past decade in many important problems of dynamics of nonlinear optical waves since the seminal works performed in 1999 [1]. Such few-cycle pulses [2,3] find applications in a wide variety of research areas such as light-matter interactions at high field intensities, high-order harmonic generation, extreme nonlinear optics [4], and attosecond physics [5,6]; see also a recent special issue dedicated to the 10th anniversary of the generation of both attosecond pulse trains and of isolated attosecond pulses [7] using high-harmonic generation processes [8].

In parallel to the fast experimental progress in the study of the formation of few-cycle pulses (FCPs) in nonlinear optical media new theoretical approaches to model their propagation in a lot of physical settings were developed. It is to be noticed that three classes of main dynamical models for FCPs have been put forward in the past years: (i) the quantum approach [9-12], (ii) the refinements within the framework of slowly varying envelope approximation (SVEA) of the nonlinear Schrödinger-type envelope equations [13-20], and the non-SVEA models [21-35]. It was therefore found in these earlier studies that the propagation of FCPs in Kerr-type nonlinear optical media can be described beyond the SVEA by using the modified Korteweg-de Vries (mKdV) [27,28], sine-Gordon (sG) [29,30], or mKdV-sG equations [31-33]. It is to be noticed that the $\mathrm{mKdV}$ and $\mathrm{sG}$ equations are completely integrable by means of the inverse scattering transform method [36,37], whereas the mKdV-sG equation is completely integrable only if some condition between its coefficients is satisfied [38,39].

We also mention other relevant recent works on the formation and dynamics of few-cycle pulses in a variety of physical settings: the propagation and interaction of extremely short electromagnetic pulses in quadratic nonlinear media [40-42], the study of few-cycle light bullets created by femtosecond filaments [43], the investigation of ultrashort spatiotemporal optical solitons in quadratic nonlinear media [44], the ultrashort spatiotemporal optical pulse propagation in cubic media without the use of the slowly varying envelope approximation $[45,46]$, the possibility of generating few-cycle dissipative optical solitons [47,48], the generation of unipolar pulses from nonunipolar optical pulses in quadratic nonlinear media [49], and the existence of guided optical solitons of femtosecond duration and nanoscopic mode area [50].

Other recent relevant studies deal with the problem of ultrafast pulse propagation in mode-locked laser cavities in the few femtosecond pulse regime and the derivation of a master mode-locking equation for ultrashort pulses [51]. Also, a recent theoretical work presents a class of few-cycle elliptically polarized solitary waves in isotropic Kerr media [52]. Moreover, robust circularly polarized few-optical-cycle solitons in Kerr media in both long-wave and short-wave approximation regimes were also studied in recent papers [53-55].

It is to be mentioned that most of theoretical investigations concern only FCPs propagating in nonlinear optical media described by a Hamiltonian related to two-level atoms. However, in a recent work [56] we extended the existing studies to a more general physical situation when we considered a multilevel system in the framework of the reductive perturbation method (multiscale analysis) [57]. In Ref. [56] we gave a detailed mathematical derivation of the $\mathrm{mKdV}$ equation for a general Hamiltonian. We assumed that the absorption spectrum of the nonlinear medium does not extend below some cutoff frequency, and that the typical frequency of the FCP is much less than the latter. In other words, we assumed that the transparency range of the medium is very large, and we considered only the frequencies located in the ultraviolet spectral domain and further; thus a nonlinear cubic (Kerr-like) medium which has no transition in the infrared was actually described in Ref. [56].

It is well known that nonlinear propagation of few-opticalcycle pulses in quadratic nonlinear optical media can be described by means of a KdV model, which can be derived in a rigorous way by means of the reductive perturbation (or multiple scales) method, either from a quantum mechanical two-level model with a nonzero dipolar momentum in the excited state, or from a classical model of anharmonic oscillators [42]. 
In the present work we intend to give a detailed mathematical derivation of the coupled $\mathrm{KdV}$ equations describing ultrashort soliton propagation in quadratic nonlinear media by considering a general Hamiltonian for multilevel atoms. We thus assume that the absorption spectrum of the medium does not extend below some cutoff frequency, and that the typical frequency of the FCP is much less than the latter. We therefore consider that the transparency range of the medium is very large, and we take into account only the frequencies located in the ultraviolet domain and further. The effect of the infrared transitions, which yield a sG model in the case of two-level atoms and cubic nonlinearity, will be considered in a further study.

This paper is organized as follows: In the next section we present in detail the governing Heisenberg equations for the density matrix $\rho$ in the case of the most general Hamiltonian for multilevel atoms and we analyze the corresponding Maxwell wave equations. We consider the case of quadratic nonlinear media (i.e., we assume a noncentrosymmetric material with a nonvanishing second-order susceptibility $\left.\chi^{(2)}\right)$. We work in the so-called long-wave regime and we perform the multiscale analysis [57] order by order. In Sec. III we analyze in detail the nondegenerate case (i.e., we assume that the two values of the refractive indices are distinct). Then, in Sec. IV we consider the degenerate case, i.e., we consider that the two possible linear polarizations propagating in the direction defined by the $z$ axis have the same refractive index $n$. In the particular case when the propagation axis $z$ is one of the eigenaxes of the medium, the degeneracy conditions imply that the crystal is uniaxial and the optical axis is the propagation one. The study of linear eigenpolarizations in the degenerate case and the corresponding formation of half-cycle solitons from few-cycle inputs are given in Sec. V. Finally, in Sec. VI we present our conclusions.

\section{STARTING GOVERNING EQUATIONS AND MULTISCALE ANALYSIS}

We consider a set of atoms described by a very general Hamiltonian:

$$
\hat{H}_{0}=\hbar\left(\begin{array}{cccc}
\omega_{1} & 0 & \cdots & 0 \\
0 & \omega_{2} & \cdots & \cdots \\
\cdots & \cdots & \cdots & \cdots \\
0 & \cdots & \cdots & \omega_{N}
\end{array}\right)
$$

The evolution of the density matrix $\rho$ is governed by the Heisenberg equation

$$
i \hbar \partial_{t} \rho=[\hat{H}, \rho]
$$

in which the total Hamiltonian

$$
\hat{H}=\hat{H}_{0}-\vec{\mu} \cdot \vec{E}
$$

includes a term accounting for the coupling between the electric field $\vec{E}$ and the atoms through a dipolar momentum operator $\vec{\mu}=\left(\mu^{x}, \mu^{y}, \mu^{z}\right)$, in which the matrices

$$
\mu^{\alpha}=\left(\mu_{m n}^{\alpha}\right)_{(m n) \in[1, N]}
$$

are Hermitian (i.e., $\mu_{n m}^{\alpha}=\mu_{m n}^{\alpha}{ }^{*}$, where the star denotes the complex conjugate), and $\alpha=x, y, z$.
The evolution of the electric field is governed by the Maxwell wave equation

$$
\vec{\nabla}^{2} \vec{E}-\vec{\nabla}(\vec{\nabla} \cdot \vec{E})=\frac{1}{c^{2}} \partial_{t}^{2}\left(\vec{E}+\frac{1}{\epsilon_{0}} \vec{P}\right),
$$

in which $\vec{\nabla}$ is the gradient operator, and the polarization density $\vec{P}$ is expressed as

$$
\vec{P}=N \operatorname{Tr}(\rho \vec{\mu}) .
$$

We consider a quadratic nonlinearity; that is, we assume that the material is noncentrosymmetric, so that the second-order susceptibility $\chi^{(2)}$ does not vanish.

We introduce the slow variables

$$
\begin{gathered}
\tau=\varepsilon\left(t-\frac{z}{V}\right), \\
\zeta=\varepsilon^{3} t,
\end{gathered}
$$

so that

$$
\begin{gathered}
\partial_{t}=\varepsilon \partial_{\tau}, \\
\partial_{z}=-\frac{\varepsilon}{V} \partial_{\tau}+\varepsilon^{3} \partial_{\zeta} .
\end{gathered}
$$

The electric field $\vec{E}$, the polarization density $\vec{P}$ and the density matrix $\rho$ are expanded in a power series of some small parameter $\varepsilon$ as [57]

$$
\begin{gathered}
\vec{E}=\sum_{p \geqslant 2} \varepsilon^{p} \vec{E}_{p}=\sum_{p \geqslant 2} \varepsilon^{p}\left(u_{p}, v_{p}, w_{p}\right) \\
\vec{P}=\sum_{p \geqslant 2} \varepsilon^{p} \vec{P}_{p}=\sum_{p \geqslant 2} \varepsilon^{p}\left(P_{p}, Q_{p}, R_{p}\right) \\
\rho=\sum_{p \geqslant 0} \varepsilon^{p} \rho^{(p)} .
\end{gathered}
$$

\section{A. Order 0}

The Heisenberg equation (2) at order $\varepsilon^{0}$ is

$$
0=\left[\hat{H}_{0}, \rho^{(0)}\right] \text {, }
$$

and since

$$
\left(\left[H_{0}, \rho\right]\right)_{m n}=\hbar \Omega_{m n} \rho_{m n},
$$

in which we have set

$$
\Omega_{m n}=\omega_{m}-\omega_{n},
$$

it yields $\rho_{m n}^{(0)}=0$ for $m \neq n(m, n=1,2, \ldots, N)$.

\section{B. Order 1}

The Heisenberg equation (2) at order $\varepsilon^{1}$ is

$$
i \hbar \partial_{\tau} \rho^{(0)}=\left[\hat{H}_{0}, \rho^{(1)}\right] .
$$

Using Eq. (15) into Eq. (17), we obtain the equations

$$
i \hbar \partial_{\tau} \rho_{m m}^{(0)}=0
$$

and

$$
i \hbar \partial_{\tau} \rho_{m n}^{(0)}=-\hbar \Omega_{m n} \rho_{m n}^{(1)}
$$


for $m \neq n$. Consequently, $\rho_{m n}^{(1)}=0$ for $m \neq n$, while the diagonal terms $\rho_{m m}^{(0)}$ are constant.

\section{Order 2}

The Heisenberg equation (2) at order $\varepsilon^{2}$ is

$$
i \hbar \partial_{\tau} \rho^{(1)}=\left[\hat{H}_{0}, \rho^{(2)}\right]-\vec{E}_{2} \cdot\left[\vec{\mu}, \rho^{(0)}\right] .
$$

Since

$$
\left(\left[\mu^{\alpha}, \rho\right]\right)_{m n}=\sum_{\nu=1}^{N}\left(\mu_{m v}^{\alpha} \rho_{\nu n}-\rho_{m v} \mu_{v n}^{\alpha}\right),
$$

and using (15), we obtain the equations

$$
\begin{aligned}
i \hbar \partial_{\tau} \rho_{m n}^{(1)}= & \hbar \Omega_{m n} \rho_{m n}^{(2)}-u_{2} \sum_{\nu=1}^{N}\left(\mu_{m \nu}^{x} \rho_{v n}^{(0)}-\rho_{m \nu}^{(0)} \mu_{\nu n}^{x}\right) \\
& -v_{2} \sum_{\nu=1}^{N}\left(\mu_{m \nu}^{y} \rho_{v n}^{(0)}-\rho_{m \nu}^{(0)} \mu_{v n}^{y}\right) \\
& -w_{2} \sum_{\nu=1}^{N}\left(\mu_{m \nu}^{z} \rho_{\nu n}^{(0)}-\rho_{m \nu}^{(0)} \mu_{v n}^{z}\right)
\end{aligned}
$$

for $m \neq n$ and

$$
\begin{aligned}
i \hbar \partial_{\tau} \rho_{m m}^{(1)}= & -u_{2} \sum_{n=1}^{N}\left(\mu_{m n}^{x} \rho_{n m}^{(0)}-\rho_{m n}^{(0)} \mu_{n m}^{x}\right) \\
& -v_{2} \sum_{n=1}^{N}\left(\mu_{m n}^{y} \rho_{n m}^{(0)}-\rho_{m n}^{(0)} \mu_{n m}^{y}\right) \\
& -w_{2} \sum_{n=1}^{N}\left(\mu_{m n}^{z} \rho_{n m}^{(0)}-\rho_{m n}^{(0)} \mu_{n m}^{z}\right) .
\end{aligned}
$$

The off-diagonal components of both $\rho^{(0)}$ and $\rho^{(1)}$ being zero, Eq. (22) reduces to

$$
\rho_{m n}^{(2)}=\frac{\left(\rho_{n n}^{(0)}-\rho_{m m}^{(0)}\right)}{\hbar \Omega_{m n}}\left(u_{2} \mu_{m n}^{x}+v_{2} \mu_{m n}^{y}+w_{2} \mu_{m n}^{z}\right)
$$

while Eq. (23) shows that the diagonal components $\rho_{m m}^{(1)}$ should be constant. We assume that $\rho^{(0)}$ tends to its thermal equilibrium value, and all $\rho^{p}$ with $p \geqslant 1$ tend to zero, at infinity, and consequently $\rho_{m m}^{(1)}=0$ for any $m$.

The polarization density $\vec{P}$ is given by Eq. (6), which yields at order $\varepsilon^{2}$

$$
P_{2}=N \sum_{n m} \rho_{n m}^{(2)} \mu_{m n}
$$

The diagonal terms $\rho_{m m}^{(2)}$ are zero. This point will be presented below with the third order for the sake of clarity, but can be derived now, and the sum in Eq. (25) extends on $m \neq n$ only. Then, using the results of order 1 , we get

$$
P_{2}=N \sum_{n m} \frac{\left(u_{2} \mu_{n m}^{x}+v_{2} \mu_{n m}^{y}+w_{2} \mu_{n m}^{z}\right)\left(\rho_{m m}^{(0)}-\rho_{n n}^{(0)}\right)}{\hbar \Omega_{n m}} \mu_{m n}^{x},
$$

etc., which can be rewritten as

$$
\vec{P}_{2}=\epsilon_{0} \chi^{(1)} \cdot \vec{E}_{2},
$$

where $\chi^{(1)}$ is the linear susceptibility tensor given by

$$
\chi_{\alpha \beta}^{(1)}=\frac{N}{\epsilon_{0} \hbar} \sum_{n m}\left(\rho_{m m}^{(0)}-\rho_{n n}^{(0)}\right) \frac{\mu_{n m}^{\alpha} \mu_{m n}^{\beta}}{\Omega_{n m}},
$$

where $\alpha, \beta=x, y, z$. Equation (28) is worth being compared to the known expression of the linear susceptibility, which is (Eq. 3.5.15, p. 163 of Ref. [58])

$$
\chi_{\alpha \beta}^{(1)}(\omega)=\frac{N}{\epsilon_{0} \hbar} \sum_{n m}\left(\rho_{m m}^{(0)}-\rho_{n n}^{(0)}\right) \frac{\mu_{n m}^{\alpha} \mu_{m n}^{\beta}}{\left(\Omega_{n m}-\omega\right)-i \gamma_{n m}} .
$$

Here we neglect the damping (i.e., $\gamma_{n m}=0$ ) and, due to the long-wave approximation, the susceptibility must be evaluated as $\omega$ tends to zero. Then Eq. (28) coincides with Eq. (29).

Now let us consider the Maxwell wave equation (5) at the leading order $\varepsilon^{4}$ :

$$
\begin{gathered}
\frac{1}{V^{2}} \partial_{\tau}^{2} u_{2}=\frac{1}{c^{2}} \partial_{\tau}^{2}\left(u_{2}+\frac{1}{\epsilon_{0}} P_{2}\right), \\
\frac{1}{V^{2}} \partial_{\tau}^{2} v_{2}=\frac{1}{c^{2}} \partial_{\tau}^{2}\left(v_{2}+\frac{1}{\epsilon_{0}} Q_{2}\right), \\
0=\frac{1}{c^{2}} \partial_{\tau}^{2}\left(w_{2}+\frac{1}{\epsilon_{0}} R_{2}\right) .
\end{gathered}
$$

By substituting Eq. (27) into Eqs. (30)-(32) we get, after two integrations with respect to $\tau$,

$$
\begin{gathered}
n^{2} u_{2}=u_{2}+\left(\chi_{x x}^{(1)} u_{2}+\chi_{x y}^{(1)} v_{2}+\chi_{x z}^{(1)} w_{2}\right), \\
n^{2} v_{2}=v_{2}+\left(\chi_{y x}^{(1)} u_{2}+\chi_{y y}^{(1)} v_{2}+\chi_{y z}^{(1)} w_{2}\right), \\
0=w_{2}+\left(\chi_{z x}^{(1)} u_{2}+\chi_{z y}^{(1)} v_{2}+\chi_{z z}^{(1)} w_{2}\right),
\end{gathered}
$$

where $n=c / V$ is the refractive index. Equation (35) has the solution

$$
w_{2}=-S_{x} u_{2}-S_{y} v_{2},
$$

where we have set

$$
S_{\alpha}=\frac{\chi_{\alpha z}^{(1)}}{1+\chi_{z z}^{(1)}},
$$

for $\alpha=x, y$. Then Eqs. (33) and (34) become

$$
\begin{aligned}
& \left(1-n^{2}+\chi_{x x}^{\prime}\right) u_{2}+\chi_{x y}^{\prime} v_{2}=0, \\
& \chi_{x y}^{\prime} u_{2}+\left(1-n^{2}+\chi_{y y}^{\prime}\right) u_{2}=0,
\end{aligned}
$$

in which we have set

$$
\chi_{\alpha \beta}^{\prime}=\chi_{\alpha \beta}^{(1)}-\frac{\chi_{\alpha z}^{(1)} \chi_{\beta z}^{(1)}}{1+\chi_{z z}^{(1)}},
$$

for $\alpha, \beta=x, y$. Recall that the tensor $\chi^{(1)}$ is real symmetrical, then the system (38), (39) has a nonzero solution if

$$
\left(1-n^{2}\right)^{2}+\left(1-n^{2}\right)\left(\chi_{x x}^{\prime}+\chi_{y y}^{\prime}\right)+\chi_{x x}^{\prime} \chi_{y y}^{\prime}-\chi_{x y}^{\prime 2}=0 .
$$


The discriminant of this second-order polynomial equation for $\left(1-n^{2}\right)$ reduces to

$$
\Delta=\left(\chi_{x x}^{\prime}-\chi_{y y}^{\prime}\right)^{2}+4 \chi_{x y}^{\prime 2},
$$

and consequently there are always two real solutions, and two distinct values of the refractive index $n$, except if $\chi_{x x}^{\prime}=\chi_{y y}^{\prime}$ and $\chi_{x y}^{\prime}=0$ [this analysis proves that $\left(1-n^{2}\right)$ is real but the condition $\left(1-n^{2}\right)<0$, which must be satisfied in real materials for physical reasons, does not follow from it].

\section{NONDEGENERATE CASE}

Let us assume that the two values of the refractive index $n$ are distinct. According to Eqs. (38) and (39), the two values of $\left(n^{2}-1\right)$ are the eigenvalues of the matrix

$$
\left(\begin{array}{ll}
\chi_{x x}^{\prime} & \chi_{x y}^{\prime} \\
\chi_{x y}^{\prime} & \chi_{y y}^{\prime}
\end{array}\right)
$$

which is a real symmetrical one and consequently admits orthogonal real eigenvectors. The mapping in the space which maps the axes $x$ and $y$ to the eigenaxes and leaves $z$ unchanged is a rotation around the $z$ axis, which is the propagation direction. Hence, by means of an adequate rotation around this axis, we can restrict to the case where $\chi_{x y}^{\prime}=0$ without loss of generality. Obviously, considering the particular case in which the propagation direction itself is an eigenaxis of the linear susceptibility $\chi^{(1)}$, would considerably simplify the analysis. However, $\chi^{(2)}$ crystals must be noncentrosymmetric, and hence they are, in general, strongly anisotropic from the point of view of nonlinear optics. It is an important point that our analysis is valid for any propagation direction in the crystal, and not only for propagation along one of the eigenaxes.

The refractive index is then given either by $n^{2}=1+\chi_{x x}^{\prime}$ or by $n^{2}=1+\chi_{y y}^{\prime}$, let us choose the first value, the refractive index is thus given by

$$
n^{2}=1+\left(\chi_{x x}^{(1)}-\frac{\left(\chi_{x z}^{(1)}\right)^{2}}{1+\chi_{z z}^{(1)}}\right) .
$$

Equations (38) and (39) then reduce to $v_{2}=0$, while $u_{2}$ remains free. Equation (36) reduces to $w_{2}=-S_{x} u_{2}$, with $S_{x}$ given by Eq. (37), which completes the analysis at this order.

\section{A. Order 3}

The Heisenberg equation (2) at order $\varepsilon^{3}$ is

$$
i \hbar \partial_{\tau} \rho^{(2)}=\left[H_{0}, \rho_{3}\right]-\vec{E}_{3} \cdot\left[\vec{\mu}, \rho^{(0)}\right]-\vec{E}_{2} \cdot\left[\vec{\mu}, \rho^{(1)}\right] .
$$

Using Eqs. (15) and (21), it yields the equations

$$
\begin{aligned}
i \hbar \partial_{\tau} \rho_{m n}^{(2)}= & \hbar \Omega_{m n} \rho_{m n}^{(3)}-u_{3} \sum_{\nu=1}^{N}\left(\mu_{m \nu}^{x} \rho_{v n}^{(0)}-\rho_{m \nu}^{(0)} \mu_{\nu n}^{x}\right)-v_{3} \sum_{\nu=1}^{N}\left(\mu_{m \nu}^{y} \rho_{v n}^{(0)}-\rho_{m \nu}^{(0)} \mu_{v n}^{y}\right)-w_{3} \sum_{\nu=1}^{N}\left(\mu_{m \nu}^{z} \rho_{v n}^{(0)}-\rho_{m \nu}^{(0)} \mu_{v n}^{z}\right) \\
& -u_{2} \sum_{\nu=1}^{N}\left(\mu_{m \nu}^{x} \rho_{v n}^{(1)}-\rho_{m \nu}^{(1)} \mu_{v n}^{x}\right)-w_{2} \sum_{\nu=1}^{N}\left(\mu_{m \nu}^{z} \rho_{v n}^{(1)}-\rho_{m \nu}^{(1)} \mu_{v n}^{z}\right)
\end{aligned}
$$

for $m \neq n$ and

$$
\begin{aligned}
i \hbar \partial_{\tau} \rho_{m m}^{(2)}= & -u_{3} \sum_{\nu=1}^{N}\left(\mu_{m \nu}^{x} \rho_{v m}^{(0)}-\rho_{m \nu}^{(0)} \mu_{v m}^{x}\right)-v_{3} \sum_{\nu=1}^{N}\left(\mu_{m \nu}^{y} \rho_{v m}^{(0)}-\rho_{m \nu}^{(0)} \mu_{v m}^{y}\right)-w_{3} \sum_{\nu=1}^{N}\left(\mu_{m \nu}^{z} \rho_{v m}^{(0)}-\rho_{m \nu}^{(0)} \mu_{v m}^{z}\right) \\
& -u_{2} \sum_{\nu=1}^{N}\left(\mu_{m \nu}^{x} \rho_{v m}^{(1)}-\rho_{m \nu}^{(1)} \mu_{v m}^{x}\right)-w_{2} \sum_{\nu=1}^{N}\left(\mu_{m \nu}^{z} \rho_{v m}^{(1)}-\rho_{m \nu}^{(1)} \mu_{v m}^{z}\right) .
\end{aligned}
$$

We used $v_{2}=0$. Since $\rho^{(0)}$ is diagonal and $\rho^{(1)}=(0)$, Eq. (46) shows that $\rho_{m m}^{(2)}=0$ for all $m$, which was used in the computation of $\vec{P}_{2}$ above. In the same way, Eq. (45) gives

$$
\rho_{m n}^{(3)}=\frac{i}{\Omega_{m n}} \partial_{\tau} \rho_{m n}^{(2)}+\frac{\left(u_{3} \mu_{m n}^{x}+v_{3} \mu_{m n}^{y}+w_{3} \mu_{m n}^{z}\right)\left(\rho_{n n}^{(0)}-\rho_{m m}^{(0)}\right)}{\hbar \Omega_{m n}}
$$

or, substituting Eq. (24) into Eq. (47) and taking into account that $w_{2}=-S_{x} u_{2}$,

$$
\rho_{m n}^{(3)}=\frac{i\left(\rho_{n n}^{(0)}-\rho_{m m}^{(0)}\right)}{\hbar \Omega_{m n}{ }^{2}}\left(\mu_{m n}^{x}-S_{x} \mu_{m n}^{z}\right) \partial_{\tau} u_{2}+\frac{\left(u_{3} \mu_{m n}^{x}+v_{3} \mu_{m n}^{y}+w_{3} \mu_{m n}^{z}\right)\left(\rho_{n n}^{(0)}-\rho_{m m}^{(0)}\right)}{\hbar \Omega_{m n}} .
$$

It allows us to obtain the polarization density at order $\varepsilon^{3}$ as

$$
P_{3}=\frac{N}{\hbar} \sum_{n m} \frac{i\left(\rho_{m m}^{(0)}-\rho_{n n}^{(0)}\right) \mu_{m n}^{x}}{\Omega_{n m}{ }^{2}}\left(\mu_{n m}^{x}-S_{x} \mu_{n m}^{z}\right) \partial_{\tau} u_{2}+\frac{N}{\hbar} \sum_{n m} \frac{\left(\rho_{m m}^{(0)}-\rho_{n n}^{(0)}\right) \mu_{m n}^{x}}{\Omega_{n m}}\left(u_{3} \mu_{n m}^{x}+v_{3} \mu_{n m}^{y}+w_{3} \mu_{n m}^{z}\right)
$$

and analogous expressions for $Q_{3}$ and $R_{3}$. Let us set

$$
A_{\alpha \beta}=\frac{N}{\epsilon_{0} \hbar} \sum_{n m} \frac{i\left(\rho_{m m}^{(0)}-\rho_{n n}^{(0)}\right) \mu_{n m}^{\alpha} \mu_{m n}^{\beta}}{\Omega_{n m}^{2}},
$$


for $\alpha, \beta=x, y, z$. It is straightforward to prove that the matrix $\left(A_{\alpha \beta}\right)_{\alpha, \beta=x, y, z}$ is skew-symmetric and real. In fact it is zero, this can be justified as follows on a physical basis: Taking the derivative of Eq. (29) (where we have set $\gamma_{n m}=0$ ), we see that

$$
A_{\alpha \beta}=\left.i \frac{d \chi_{\alpha \beta}^{(1)}(\omega)}{d \omega}\right|_{\omega=0}
$$

We assume that the decay rates $\gamma_{n m}$ are zero, and consequently no absorption occurs. In this case the linear susceptibility $\chi^{(1)}(\omega)$ must be real for any $\omega$, and so must be its derivative. Since direct computation shows that $d \chi_{\alpha \beta}^{(1)}(\omega) /\left.d \omega\right|_{\omega=0}$ is purely imaginary, we see that it must be zero (i.e., $A_{\alpha \beta}=0$ for all $\alpha, \beta)$. Then the polarization density at this order reduces to

$$
\vec{P}_{3}=\epsilon_{0} \chi^{(1)} \cdot \vec{E}_{3},
$$

with $\chi^{(1)}$ defined according to Eq. (28). The Maxwell wave equation (5) at order $\varepsilon^{5}$ is

$$
\begin{gathered}
\frac{1}{V^{2}} \partial_{\tau}^{2} u_{3}=\frac{1}{c^{2}} \partial_{\tau}^{2}\left(u_{3}+\frac{1}{\epsilon_{0}} P_{3}\right), \\
\frac{1}{V^{2}} \partial_{\tau}^{2} v_{3}=\frac{1}{c^{2}} \partial_{\tau}^{2}\left(v_{3}+\frac{1}{\epsilon_{0}} Q_{3}\right), \\
0=\frac{1}{c^{2}} \partial_{\tau}^{2}\left(w_{3}+\frac{1}{\epsilon_{0}} R_{3}\right) .
\end{gathered}
$$

It is thus the same system as was satisfied by $\vec{E}_{2}$. Hence its solution is straightforward: $w_{3}=-S_{x} u_{3}, v_{3}=0$, and $u_{3}$ remains free.

\section{B. Order 4}

The Heisenberg equation (2) at order $\varepsilon^{4}$ is

$$
i \hbar \partial_{\tau} \rho_{3}=\left[\hat{H}_{0}, \rho_{4}\right]-\vec{E}_{4} \cdot\left[\vec{\mu}, \rho^{(0)}\right]-\vec{E}_{3} \cdot\left[\vec{\mu}, \rho^{(1)}\right]-\vec{E}_{2} \cdot\left[\vec{\mu}, \rho^{(2)}\right] .
$$

Using Eqs. (15) and (21) into Eq. (56), we obtain the equations

$$
\begin{aligned}
& i \hbar \partial_{\tau} \rho_{m n}^{(3)}=\hbar \Omega_{m n} \rho_{m n}^{(4)}-u_{4} \sum_{\nu=1}^{N}\left(\mu_{m \nu}^{x} \rho_{\nu n}^{(0)}-\rho_{m \nu}^{(0)} \mu_{\nu n}^{x}\right)-v_{4} \sum_{\nu=1}^{N}\left(\mu_{m \nu}^{y} \rho_{\nu n}^{(0)}-\rho_{m \nu}^{(0)} \mu_{v n}^{y}\right)-w_{4} \sum_{\nu=1}^{N}\left(\mu_{m \nu}^{z} \rho_{v n}^{(0)}-\rho_{m \nu}^{(0)} \mu_{\nu n}^{z}\right) \\
& -u_{3} \sum_{\nu=1}^{N}\left(\mu_{m \nu}^{x} \rho_{\nu n}^{(1)}-\rho_{m \nu}^{(1)} \mu_{v n}^{x}\right)-w_{3} \sum_{\nu=1}^{N}\left(\mu_{m \nu}^{z} \rho_{\nu n}^{(1)}-\rho_{m \nu}^{(1)} \mu_{v n}^{z}\right) \\
& -u_{2} \sum_{\nu=1}^{N}\left(\mu_{m \nu}^{x} \rho_{\nu n}^{(2)}-\rho_{m \nu}^{(2)} \mu_{v n}^{x}\right)-w_{2} \sum_{\nu=1}^{N}\left(\mu_{m \nu}^{z} \rho_{\nu n}^{(2)}-\rho_{m \nu}^{(2)} \mu_{v n}^{z}\right)
\end{aligned}
$$

for $m \neq n$ and

$$
\begin{aligned}
& i \hbar \partial_{\tau} \rho_{m m}^{(3)}=-u_{4} \sum_{\nu=1}^{N}\left(\mu_{m \nu}^{x} \rho_{\nu m}^{(0)}-\rho_{m \nu}^{(0)} \mu_{\nu m}^{x}\right)-v_{4} \sum_{\nu=1}^{N}\left(\mu_{m \nu}^{y} \rho_{\nu m}^{(0)}-\rho_{m \nu}^{(0)} \mu_{\nu m}^{y}\right)-w_{4} \sum_{\nu=1}^{N}\left(\mu_{m \nu}^{z} \rho_{\nu m}^{(0)}-\rho_{m \nu}^{(0)} \mu_{\nu m}^{z}\right) \\
& -u_{3} \sum_{\nu=1}^{N}\left(\mu_{m \nu}^{x} \rho_{\nu m}^{(1)}-\rho_{m \nu}^{(1)} \mu_{\nu m}^{x}\right)-w_{3} \sum_{\nu=1}^{N}\left(\mu_{m \nu}^{z} \rho_{\nu m}^{(1)}-\rho_{m \nu}^{(1)} \mu_{\nu m}^{z}\right) \\
& -u_{2} \sum_{\nu=1}^{N}\left(\mu_{m v}^{x} \rho_{\nu m}^{(2)}-\rho_{m \nu}^{(2)} \mu_{v m}^{x}\right)-w_{2} \sum_{\nu=1}^{N}\left(\mu_{m \nu}^{z} \rho_{\nu m}^{(2)}-\rho_{m \nu}^{(2)} \mu_{v m}^{z}\right) \text {. }
\end{aligned}
$$

$\rho^{(0)}$ and $\rho^{(1)}$ being diagonal, Eq. (57) reduces to

$$
\begin{aligned}
i \hbar \partial_{\tau} \rho_{m n}^{(3)}= & \hbar \Omega_{m n} \rho_{m n}^{(4)}-u_{4} \mu_{m n}^{x}\left(\rho_{n n}^{(0)}-\rho_{m m}^{(0)}\right)-v_{4} \mu_{m n}^{y}\left(\rho_{n n}^{(0)}-\rho_{m m}^{(0)}\right) \\
& -w_{4} \mu_{m n}^{z}\left(\rho_{n n}^{(0)}-\rho_{m m}^{(0)}\right)-u_{3} \mu_{m n}^{x}\left(\rho_{n n}^{(1)}-\rho_{m m}^{(1)}\right)-w_{3} \mu_{m n}^{z}\left(\rho_{n n}^{(1)}-\rho_{m m}^{(1)}\right) \\
& -u_{2} \sum_{\nu=1}^{N}\left(\mu_{m v}^{x} \rho_{v n}^{(2)}-\rho_{m v}^{(2)} \mu_{v n}^{x}\right)-w_{2} \sum_{\nu=1}^{N}\left(\mu_{m v}^{z} \rho_{v n}^{(2)}-\rho_{m v}^{(2)} \mu_{v n}^{z}\right) .
\end{aligned}
$$

Then substituting Eqs. (24) and (48) into Eq. (59) yields

$$
\begin{aligned}
\rho_{m n}^{(4)}= & \frac{i}{\Omega_{m n}} \partial_{\tau} \rho_{m n}^{(3)}+\frac{\left(\rho_{n n}^{(0)}-\rho_{m m}^{(0)}\right)}{\hbar \Omega_{m n}}\left(u_{4} \mu_{m n}^{x}+v_{4} \mu_{m n}^{y}+w_{4} \mu_{m n}^{z}\right)+\frac{\left(\rho_{n n}^{(1)}-\rho_{m m}^{(1)}\right)}{\hbar \Omega_{m n}}\left(\mu_{m n}^{x}-S_{x} \mu_{m n}^{z}\right) u_{3} \\
& +\frac{1}{\hbar \Omega_{m n}}\left[\sum_{\nu=1}^{N}\left(\mu_{m v}^{x} \rho_{v n}^{(2)}-\rho_{m v}^{(2)} \mu_{v n}^{x}\right)-S_{x} \sum_{\nu=1}^{N}\left(\mu_{m v}^{z} \rho_{v n}^{(2)}-\rho_{m v}^{(2)} \mu_{v n}^{z}\right)\right] u_{2} .
\end{aligned}
$$


The corresponding polarization density is

$$
\begin{aligned}
P_{4}= & -\frac{N}{\hbar} \sum_{n m} \frac{\left(\rho_{m m}^{(0)}-\rho_{n n}^{(0)}\right) \mu_{m n}^{x}}{\Omega_{n m}^{3}}\left(\mu_{n m}^{x}-S_{x} \mu_{n m}^{z}\right) \partial_{\tau}^{2} u_{2}+\frac{N}{\hbar} \sum_{n m} \frac{i\left(\rho_{m m}^{(0)}-\rho_{n n}^{(0)}\right) \mu_{m n}^{x}}{\Omega_{n m}{ }^{2}}\left(\mu_{n m}^{x}-S_{x} \mu_{n m}^{z}\right) \partial_{\tau} u_{3} \\
& +\frac{N}{\hbar} \sum_{n m} \frac{\left(\rho_{m m}^{(0)}-\rho_{n n}^{(0)}\right)}{\Omega_{n m}} \mu_{m n}^{x}\left(u_{4} \mu_{n m}^{x}+v_{4} \mu_{n m}^{y}+w_{4} \mu_{n m}^{z}\right)+\frac{N}{\hbar^{2}} \sum_{n m v}\left[\frac{\left(\rho_{m m}^{(0)}-\rho_{v v}^{(0)}\right)}{\Omega_{n m} \Omega_{v m}}-\frac{\left(\rho_{v v}^{(0)}-\rho_{n n}^{(0)}\right)}{\Omega_{n m} \Omega_{n v}}\right] \mu_{m n}^{x} \mu_{v m}^{x} \mu_{n v}^{x} u_{2}{ }^{2} \\
& +\frac{N}{\hbar^{2}} \sum_{n m v}\left[\frac{\left(\rho_{m m}^{(0)}-\rho_{v v}^{(0)}\right)}{\Omega_{n m} \Omega_{v m}}-\frac{\left(\rho_{\nu v}^{(0)}-\rho_{n n}^{(0)}\right)}{\Omega_{n m} \Omega_{n v}}\right] \mu_{m n}^{x} \mu_{v m}^{z} \mu_{n v}^{z} S_{x}{ }^{2} u_{2}{ }^{2} \\
& -\frac{N}{\hbar^{2}} \sum_{n m v}\left[\frac{\left(\rho_{m m}^{(0)}-\rho_{v v}^{(0)}\right)}{\Omega_{n m} \Omega_{v m}}-\frac{\left(\rho_{v v}^{(0)}-\rho_{n n}^{(0)}\right)}{\Omega_{n m} \Omega_{n v}}\right] \mu_{m n}^{x}\left(\mu_{n v}^{x} \mu_{v m}^{z}+\mu_{n v}^{z} \mu_{v m}^{x}\right) S_{x} u_{2}{ }^{2}
\end{aligned}
$$

and analogous expressions for $Q_{4}$ and $R_{4}$.

The Maxwell wave equation (5) at the order $\varepsilon^{6}$ is

$$
\begin{gathered}
\frac{1}{V^{2}} \partial_{\tau}^{2} u_{4}-\frac{2}{V} \partial_{\tau} \partial_{\zeta} u_{2}=\frac{1}{c^{2}} \partial_{\tau}^{2}\left(u_{4}+\frac{1}{\epsilon_{0}} P_{4}\right), \\
\frac{1}{V^{2}} \partial_{\tau}^{2} v_{4}-\frac{2}{V} \partial_{\tau} \partial_{\zeta} v_{2}=\frac{1}{c^{2}} \partial_{\tau}^{2}\left(v_{4}+\frac{1}{\epsilon_{0}} Q_{4}\right), \\
0=\frac{1}{c^{2}} \partial_{\tau}^{2}\left(w_{4}+\frac{1}{\epsilon_{0}} R_{4}\right) .
\end{gathered}
$$

Let us introduce a few shortcuts, the first of which is $A_{\alpha \beta}$ given by Eq. (50). Recall that it is always zero. We define

$$
B_{\alpha \beta}=\frac{N}{\epsilon_{0} \hbar} \sum_{n m} \frac{\left(\rho_{m m}^{(0)}-\rho_{n n}^{(0)}\right) \mu_{n m}^{\alpha} \mu_{m n}^{\beta}}{\Omega_{n m}^{3}}
$$

for $\alpha, \beta=x, y, z$. Permutation of $n$ and $m$ in the sum in Eq. (65) shows that $B_{\alpha \beta}=B_{\beta \alpha}$. We also will need the tensorial quantity

$$
T_{\alpha \beta \gamma}=\frac{N}{\epsilon_{0} \hbar} \sum_{n m v}\left[\frac{\left(\rho_{m m}^{(0)}-\rho_{v \nu}^{(0)}\right)}{\Omega_{n m} \Omega_{v m}}-\frac{\left(\rho_{\nu v}^{(0)}-\rho_{n n}^{(0)}\right)}{\Omega_{n m} \Omega_{n v}}\right] \mu_{m n}^{\alpha} \mu_{v m}^{\beta} \mu_{n v}^{\gamma},
$$

for $\alpha, \beta, \gamma=x, y, z$. Then using the results of previous orders and the tensors (50), (65), and (66), system (62)-(64) reduces to

$$
\begin{aligned}
& \frac{1}{V^{2}} \partial_{\tau}^{2} u_{4}-\frac{2}{V} \partial_{\tau} \partial_{\zeta} u_{2} \\
& =\frac{1}{c^{2}} \partial_{\tau}^{2}\left[\left(1+\chi_{x x}^{(1)}\right) u_{4}+\chi_{x y}^{(1)} v_{4}+\chi_{x z}^{(1)} w_{4}\right. \\
& \quad+\left(A_{x x}-S_{x} A_{x z}\right) \partial_{\tau} u_{3}-\left(B_{x x}-S_{x} B_{x z}\right) \partial_{\tau}^{2} u_{2} \\
& \left.\quad+T_{x x x} u_{2}^{2}+T_{x z z} w_{2}^{2}+\left(T_{x x z}+T_{x z x}\right) u_{2} w_{2}\right], \\
& \frac{1}{V^{2}} \partial_{\tau}^{2} v_{4}=\frac{1}{c^{2}} \partial_{\tau}^{2}\left[\chi_{x y}^{(1)} u_{4}+\left(1+\chi_{y y}^{(1)}\right) v_{4}+\chi_{y z}^{(1)} w_{4}\right. \\
& \quad+\left(A_{y x}-S_{x} A_{y z}\right) \partial_{\tau} u_{3}-\left(B_{y x}-S_{x} B_{y z}\right) \partial_{\tau}^{2} u_{2} \\
& \left.\quad+T_{y x x} u_{2}{ }^{2}+T_{y z z} w_{2}{ }^{2}+\left(T_{y x z}+T_{y z x}\right) u_{2} w_{2}\right]
\end{aligned}
$$

$$
\begin{aligned}
0= & \frac{1}{c^{2}} \partial_{\tau}^{2}\left[\chi_{z x}^{(1)} u_{4}+\chi_{z y}^{(1)} v_{4}+\left(1+\chi_{z z}^{(1)}\right) w_{4}\right. \\
& +\left(A_{z x}-S_{x} A_{z z}\right) \partial_{\tau} u_{3}-\left(B_{z x}-S_{x} B_{z z}\right) \partial_{\tau}^{2} u_{2} \\
& \left.+T_{z x x} u_{2}^{2}+T_{z z z} w_{2}^{2}+\left(T_{z x z}+T_{z z x}\right) u_{2} w_{2}\right]
\end{aligned}
$$

Since all $A_{\alpha \beta}$ are zero, the terms involving $\partial_{\tau} u_{3}$ vanish. Equation (69) is solved to yield

$$
\begin{aligned}
w_{4}= & \frac{1}{1+\chi_{z z}^{(1)}}\left[-\chi_{z x}^{(1)} u_{4}-\chi_{z y}^{(1)} v_{4}\right. \\
& \left.+\left(B_{z x}-S_{x} B_{z z}\right) \partial_{\tau}^{2} u_{2}+T_{z x x}^{\prime} u_{2}^{2}\right]
\end{aligned}
$$

where we have set

$$
T_{z x x}^{\prime}=T_{z x x}-S_{x}\left(T_{z x z}+T_{z z x}\right)+S_{x}^{2} T_{z z z} .
$$

Then, reporting $w_{4}$ into Eqs. (67) and (68), we get a system of equations for $u_{4}$ and $v_{4}$, whose homogeneous part is identical to system (38) and (39) for $u_{2}$ and $v_{2}$. Hence $v_{4}$ can be computed as a function of $u_{2}$ and $u_{3}$ in Eq. (68), while both $u_{4}$ and $v_{4}$ vanish from Eq. (67), which can be reduced to

$$
2 n c \partial_{\zeta} u_{2}-\frac{\beta_{2}}{2} \partial_{\tau}^{3} u_{2}+\Xi \partial_{\tau} u_{2}^{2}=0,
$$

which is the $\mathrm{KdV}$ equation and where we have set

$$
\Xi=T_{x x x}-S_{x}\left(T_{x x z}+T_{x z x}\right)+S_{x}^{2} T_{x z z}-S_{x} T_{z x x}^{\prime}
$$

and

$$
\beta_{2}=2\left(B_{x x}-2 S_{x} B_{x z}+S_{x}^{2} B_{z z}\right) .
$$

Taking the derivative of Eq. (29) (with $\gamma_{n m}=0$ ), we see that

$$
B_{\alpha \beta}=\left.\frac{1}{2} \frac{d^{2} \chi_{\alpha \beta}^{(1)}(\omega)}{d \omega^{2}}\right|_{\omega=0}
$$

Hence,

$$
\beta_{2}=\left.\left(1,0,-S_{x}\right) \cdot \frac{d^{2} \chi^{(1)}}{d \omega^{2}}\right|_{\omega=0} \cdot\left(\begin{array}{c}
1 \\
0 \\
-S_{x}
\end{array}\right)
$$

Computing the second derivative of $\chi_{x x}^{\prime}=n^{2}-1$ defined by Eq. (40) and taking into account the fact that the first-order 
derivatives $d \chi_{\alpha \beta}^{(1)} / d \omega=0$ for $\omega=0$ shows that

$$
\beta_{2}=\left.\frac{d^{2} n^{2}}{d \omega^{2}}\right|_{\omega=0},
$$

which gives a simple expression of the dispersion coefficient $\beta_{2}$.

The nonlinear coefficient can be reduced as follows: The expression of the second-order susceptibility $\chi^{(2)}$ can be found in Ref. [58] (Eq. 3.6.14, p. 173) as

$$
\begin{aligned}
& \chi_{\alpha \beta \gamma}^{(2)}\left(\omega_{p}+\omega_{q} ; \omega_{q}, \omega_{p}\right) \\
& =\frac{N}{2 \epsilon_{0} \hbar^{2}} \sum_{m n v}\left\{\left(\rho_{m m}^{(0)}-\rho_{v v}^{(0)}\right)\left[\frac{\mu_{m n}^{\alpha} \mu_{n v}^{\beta} \mu_{v m}^{\gamma}}{\left(\Omega_{n m}-\omega_{p}-\omega_{q}-i \gamma_{n m}\right)\left(\Omega_{v m}-\omega_{p}-i \gamma_{v m}\right)}+\frac{\mu_{m n}^{\alpha} \mu_{n v}^{\gamma} \mu_{v m}^{\beta}}{\left(\Omega_{n m}-\omega_{p}-\omega_{q}-i \gamma_{n m}\right)\left(\Omega_{v m}-\omega_{q}-i \gamma_{v m}\right)}\right]\right. \\
& \left.\quad-\left(\rho_{v v}^{(0)}-\rho_{n n}^{(0)}\right)\left[\frac{\mu_{m n}^{\alpha} \mu_{v m}^{\gamma} \mu_{n v}^{\beta}}{\left(\Omega_{n m}-\omega_{p}-\omega_{q}-i \gamma_{n m}\right)\left(\Omega_{n v}-\omega_{p}-i \gamma_{n v}\right)}+\frac{\mu_{n}}{\left(\Omega_{n m}-\omega_{p}-\omega_{q}-i \gamma_{n m}\right)\left(\Omega_{n v}-\omega_{q}-i \gamma_{n v}\right)}\right]\right\} . \quad(78)
\end{aligned}
$$

It is thus seen that

$$
\chi_{\alpha \beta \gamma}^{(2)}(0 ; 0,0)=\frac{1}{2}\left(T_{\alpha \beta \gamma}+T_{\alpha \gamma \beta}\right),
$$

where we have neglected damping $\left(\gamma_{m n}=0\right)$. Hence,

$$
\Xi=\left(1,0,-S_{x}\right) \cdot \chi_{\alpha \beta \gamma}^{(2)}(0 ; 0,0):\left(\begin{array}{c}
1 \\
0 \\
-S_{x}
\end{array}\right),\left(\begin{array}{c}
1 \\
0 \\
-S_{x}
\end{array}\right) .
$$

It is straightforwardly checked that expressions (77) and (80) of the coefficients of the $\mathrm{KdV}$ equation reduce to the expressions found in Ref. [42] in the scalar case. Hence we have proved that the KdV equation derived in Ref. [42] holds for an arbitrary number of atomic levels and arbitrary propagation direction, using in the latter case the effective refractive index and nonlinear coefficients seen by the wave. The only assumption is that (i) the transitions which contribute are all well above the wave frequency and (ii) the difference between the two indices in the chosen propagation direction cannot be neglected.

\section{DEGENERATE CASE}

We now assume that the two possible linear polarizations propagating in the direction defined by the $z$ axis have the same index of refraction $n$. Mathematically, this assumption is that Eq. (41), which is the dispersion relation, has a double root, which according to Eq. (42) implies that

$$
\chi_{x x}^{\prime}=\chi_{y y}^{\prime} \quad \text { and } \quad \chi_{x y}^{\prime}=0 .
$$

If the propagation axis $z$ is one of the eigenaxes of the material, then $\chi_{\alpha z}^{(1)}=0$ for $\alpha=x, y$, and Eq. (40) reduces to $\chi_{\alpha \beta}^{\prime}=$ $\chi_{\alpha \beta}^{(1)}$. In this particular case, the degeneracy condition is that the crystal is uniaxial, and the optical axis is the propagation axis ( $z$ axis). However, the analysis remains valid for any coincidence of the two eigenvalues of the optical index. Is it easy to prove that such a coincidence may occur in a biaxial crystal. Let us call $X, Y, Z$ the eigendirections, $n_{X}, n_{Y}, n_{Z}$ the three eigenindices, and assume $n_{X}<n_{Y}<n_{Z}$. We assume a propagation direction in the $X Z$ plane, making an angle $\theta$ with the $X$ axis. Then one of the two eigenvalues of the optical index in the propagation direction defined by $\theta$ is $n_{Y}$, while the other one-let us call it $n(\theta)$-ranges from $n_{Z}$ as $\theta=0$ to $n_{X}$ as $\theta=\pi / 2$. Since $n(\theta)$ varies continuously, $n(0)<n_{Y}$ and $n(\pi / 2)>n_{Y}$, the indices $n(\theta)$ and $n_{Y}$ must coincide for some value of $\theta$. The condition that the propagation direction lies in the $X Z$ plane is obviously required for getting a simple proof only, a coincidence will also occur for other propagation directions, not too far from the $X Z$ plane. The assumption (81) is thus physically relevant and more general than the mere assumption of propagation along the optical axis; that is why we consider it.

The set of equations (33) and (34) gotten at order $\varepsilon^{2}$ reduces thus to $w_{2}=-S_{x} u_{2}-S_{y} v_{2}$, [i.e., Eq. (36)], with $S_{x}$ and $S_{y}$ given by Eq. (37), which is the value of the refractive index given by Eq. (43), while $u_{2}$ and $v_{2}$ remain free.

\section{A. Order 3}

The Heisenberg equation (2) at order $\varepsilon^{3}$ is still Eq. (44); however, Eq. (45) for $m \neq n$ becomes

$$
\begin{aligned}
& i \hbar \partial_{\tau} \rho_{m n}^{(2)}=\hbar \Omega_{m n} \rho_{m n}^{(3)}-u_{3} \sum_{\nu=1}^{N}\left(\mu_{m \nu}^{x} \rho_{\nu n}^{(0)}-\rho_{m \nu}^{(0)} \mu_{v n}^{x}\right) \\
& -v_{3} \sum_{\nu=1}^{N}\left(\mu_{m \nu}^{y} \rho_{v n}^{(0)}-\rho_{m \nu}^{(0)} \mu_{v n}^{y}\right) \\
& -w_{3} \sum_{\nu=1}^{N}\left(\mu_{m \nu}^{z} \rho_{\nu n}^{(0)}-\rho_{m \nu}^{(0)} \mu_{\nu n}^{z}\right) \\
& -u_{2} \sum_{\nu=1}^{N}\left(\mu_{m \nu}^{x} \rho_{\nu n}^{(1)}-\rho_{m \nu}^{(1)} \mu_{v n}^{x}\right) \\
& -v_{2} \sum_{\nu=1}^{N}\left(\mu_{m \nu}^{y} \rho_{\nu n}^{(1)}-\rho_{m \nu}^{(1)} \mu_{v n}^{y}\right) \\
& +\left(S_{x} u_{2}+S_{y} v_{2}\right) \sum_{\nu=1}^{N}\left(\mu_{m v}^{z} \rho_{\nu n}^{(1)}-\rho_{m \nu}^{(1)} \mu_{\nu n}^{z}\right) .
\end{aligned}
$$

Equation (46) for the diagonal term is modified in the same way. 
As in the degenerate case, $\rho_{m m}^{(2)}=0$ for all $m$, and Eq. (47) holds. Then Eq. (48) is replaced with

$$
\rho_{m n}^{(3)}=\frac{i\left(\rho_{n n}^{(0)}-\rho_{m m}^{(0)}\right)}{\hbar\left(\Omega_{m n}\right)^{2}}\left[\left(\mu_{m n}^{x}-S_{x} \mu_{m n}^{z}\right) \partial_{\tau} u_{2}+\left(\mu_{m n}^{y}-S_{y} \mu_{m n}^{z}\right) \partial_{\tau} v_{2}\right]+\frac{\left(u_{3} \mu_{m n}^{x}+v_{3} \mu_{m n}^{y}+w_{3} \mu_{m n}^{z}\right)\left(\rho_{n n}^{(0)}-\rho_{m m}^{(0)}\right)}{\hbar \Omega_{m n}}
$$

The polarization density at order $\varepsilon^{3}$ is thus

$$
P_{3}=\epsilon_{0}\left[\left(A_{x x}-S_{x} A_{x z}\right) \partial_{\tau} u_{2}+\left(A_{x y}-S_{y} A_{x z}\right) \partial_{\tau} v_{2}\right]+\frac{N}{\hbar} \sum_{n m} \frac{\left(\rho_{m m}^{(0)}-\rho_{n n}^{(0)}\right) \mu_{m n}^{x}}{\Omega_{n m}}\left(u_{3} \mu_{n m}^{x}+v_{3} \mu_{n m}^{y}+w_{3} \mu_{n m}^{z}\right),
$$

where we used the shortcut $A_{\alpha \beta}(\alpha, \beta=x, y, z)$ given by Eq. (50). $Q_{3}$ and $R_{3}$ have analogous expressions. Since it was seen from Eq. (51) that $A_{\alpha \beta}$ is always zero, the polarization density at this order still reduces to Eq. (52), and the Maxwell wave equation at this order $\varepsilon^{5}$ [Eqs. (53)-(55)] coincides again with the same system as was satisfied by $\vec{E}_{2}$. Its solution is thus $w_{3}=-S_{x} u_{3}-S_{y} v_{3}$, where $u_{3}$ and $v_{3}$ remain free.

\section{B. Order 4}

The Heisenberg equation (2) at order $\varepsilon^{4}$ is Eq. (56) as in the nondegenerate case. Equation (57) for $m \neq n$ is modified as

$$
\begin{aligned}
& i \hbar \partial_{\tau} \rho_{m n}^{(3)}=\hbar \Omega_{m n} \rho_{m n}^{(4)}-u_{4} \sum_{\nu=1}^{N}\left(\mu_{m \nu}^{x} \rho_{\nu n}^{(0)}-\rho_{m \nu}^{(0)} \mu_{\nu n}^{x}\right)-v_{4} \sum_{\nu=1}^{N}\left(\mu_{m \nu}^{y} \rho_{\nu n}^{(0)}-\rho_{m \nu}^{(0)} \mu_{v n}^{y}\right)-w_{4} \sum_{\nu=1}^{N}\left(\mu_{m \nu}^{z} \rho_{\nu n}^{(0)}-\rho_{m \nu}^{(0)} \mu_{v n}^{z}\right) \\
& -u_{3} \sum_{\nu=1}^{N}\left(\mu_{m \nu}^{x} \rho_{\nu n}^{(1)}-\rho_{m \nu}^{(1)} \mu_{\nu n}^{x}\right)-v_{3} \sum_{\nu=1}^{N}\left(\mu_{m \nu}^{y} \rho_{\nu n}^{(1)}-\rho_{m \nu}^{(1)} \mu_{\nu n}^{y}\right)+\left(S_{x} u_{3}+S_{y} v_{3}\right) \sum_{\nu=1}^{N}\left(\mu_{m \nu}^{z} \rho_{\nu n}^{(1)}-\rho_{m \nu}^{(1)} \mu_{v n}^{z}\right) \\
& -u_{2} \sum_{\nu=1}^{N}\left(\mu_{m \nu}^{x} \rho_{v n}^{(2)}-\rho_{m \nu}^{(2)} \mu_{v n}^{x}\right)-v_{2} \sum_{\nu=1}^{N}\left(\mu_{m v}^{y} \rho_{v n}^{(2)}-\rho_{m \nu}^{(2)} \mu_{v n}^{y}\right)+\left(S_{x} u_{2}+S_{y} v_{2}\right) \sum_{\nu=1}^{N}\left(\mu_{m \nu}^{z} \rho_{\nu n}^{(2)}-\rho_{m \nu}^{(2)} \mu_{v n}^{z}\right) .
\end{aligned}
$$

Equation (58) for the diagonal terms is modified in an analogous way, which allows us to compute $\rho_{m n}^{(4)}$ as

$$
\begin{aligned}
\rho_{m n}^{(4)}= & \frac{i}{\Omega_{m n}} \partial_{\tau} \rho_{m n}^{(3)}+\frac{\left(\rho_{n n}^{(0)}-\rho_{m m}^{(0)}\right)}{\hbar \Omega_{m n}}\left(u_{4} \mu_{m n}^{x}+v_{4} \mu_{m n}^{y}+w_{4} \mu_{m n}^{z}\right)+\frac{\left(\rho_{n n}^{(1)}-\rho_{m m}^{(1)}\right)}{\hbar \Omega_{m n}}\left[\left(\mu_{m n}^{x}-S_{x} \mu_{m n}^{z}\right) u_{3}+\left(\mu_{m n}^{y}-S_{y} \mu_{m n}^{z}\right) v_{3}\right] \\
& +\frac{1}{\hbar \Omega_{m n}}\left\{\left[\sum_{\nu=1}^{N}\left(\mu_{m \nu}^{x} \rho_{v n}^{(2)}-\rho_{m v}^{(2)} \mu_{v n}^{x}\right)-S_{x} \sum_{\nu=1}^{N}\left(\mu_{m \nu}^{z} \rho_{v n}^{(2)}-\rho_{m v}^{(2)} \mu_{v n}^{z}\right)\right] u_{2}\right. \\
& \left.+\left[\sum_{\nu=1}^{N}\left(\mu_{m v}^{y} \rho_{v n}^{(2)}-\rho_{m \nu}^{(2)} \mu_{v n}^{y}\right)-S_{y} \sum_{\nu=1}^{N}\left(\mu_{m v}^{z} \rho_{v n}^{(2)}-\rho_{m v}^{(2)} \mu_{v n}^{z}\right)\right] v_{2}\right\} .
\end{aligned}
$$

The corresponding polarization density thus involves terms in $\partial_{\tau} v_{3}, \partial_{\tau}^{2} v_{2}, v_{2}^{2}$, and $u_{2} v_{2}$ in addition to those present in Eq. (61). The Maxwell wave equation (5) at the order $\varepsilon^{6}$ [Eqs. (62)-(64)] can be written down using the shortcuts $A_{\alpha \beta}, B_{\alpha \beta}, T_{\alpha \beta \gamma}$ $(\alpha, \beta, \gamma=x, y, z)$ given by Eqs. (50), (65), and (66) respectively, as

$$
\begin{aligned}
& \frac{1}{V^{2}} \partial_{\tau}^{2} u_{4}-\frac{2}{V} \partial_{\tau} \partial_{\zeta} u_{2}=\frac{1}{c^{2}} \partial_{\tau}^{2}\left[\left(1+\chi_{x x}^{(1)}\right) u_{4}+\chi_{x y}^{(1)} v_{4}+\chi_{x z}^{(1)} w_{4}+\left(A_{x x}-S_{x} A_{x z}\right) \partial_{\tau} u_{3}+\left(A_{x y}-S_{y} A_{x z}\right) \partial_{\tau} v_{3}\right. \\
& -\left(B_{x x}-S_{x} B_{x z}\right) \partial_{\tau}^{2} u_{2}-\left(B_{x y}-S_{y} B_{x z}\right) \partial_{\tau}^{2} v_{2}+T_{x x x} u_{2}^{2}+T_{x y y} v_{2}^{2}+T_{x z z} w_{2}^{2}+\left(T_{x x y}+T_{x y x}\right) u_{2} v_{2} \\
& \left.+\left(T_{x x z}+T_{x z x}\right) u_{2} w_{2}+\left(T_{x y z}+T_{x z y}\right) v_{2} w_{2}\right] \\
& \frac{1}{V^{2}} \partial_{\tau}^{2} v_{4}-\frac{2}{V} \partial_{\tau} \partial_{\zeta} v_{2}=\frac{1}{c^{2}} \partial_{\tau}^{2}\left[\chi_{y x}^{(1)} u_{4}+\left(1+\chi_{y y}^{(1)}\right) v_{4}+\chi_{y z}^{(1)} w_{4}+\left(A_{y x}-S_{x} A_{y z}\right) \partial_{\tau} u_{3}+\left(A_{y y}-S_{y} A_{y z}\right) \partial_{\tau} v_{3}\right. \\
& -\left(B_{y x}-S_{x} B_{y z}\right) \partial_{\tau}^{2} u_{2}-\left(B_{y y}-S_{y} B_{y z}\right) \partial_{\tau}^{2} v_{2}+T_{y x x} u_{2}^{2}+T_{y y y} v_{2}^{2}+T_{y z z} w_{2}^{2}+\left(T_{y x y}+T_{y y x}\right) u_{2} v_{2} \\
& \left.+\left(T_{y x z}+T_{y z x}\right) u_{2} w_{2}+\left(T_{y y z}+T_{y z y}\right) v_{2} w_{2}\right] \\
& 0=\frac{1}{c^{2}} \partial_{\tau}^{2}\left[\chi_{z x}^{(1)} u_{4}+\chi_{z y}^{(1)} v_{4}+\left(1+\chi_{z z}^{(1)}\right) w_{4}+\left(A_{z x}-S_{x} A_{z z}\right) \partial_{\tau} u_{3}+\left(A_{z y}-S_{y} A_{z z}\right) \partial_{\tau} v_{3}\right. \\
& -\left(B_{z x}-S_{x} B_{z z}\right) \partial_{\tau}^{2} u_{2}-\left(B_{z y}-S_{y} B_{z z}\right) \partial_{\tau}^{2} v_{2}+T_{z x x} u_{2}^{2}+T_{z y y} v_{2}^{2}+T_{z z z} w_{2}^{2}+\left(T_{z x y}+T_{z y x}\right) u_{2} v_{2} \\
& \left.+\left(T_{z x z}+T_{z z x}\right) u_{2} w_{2}+\left(T_{z y z}+T_{z z y}\right) v_{2} w_{2}\right] \text {. }
\end{aligned}
$$


Since all $A_{\alpha \beta}$ are zero, the terms involving $\partial_{\tau} u_{3}$ or $\partial_{\tau} v_{3}$ vanish. Equation (89) is solved to yield

$$
\begin{aligned}
w_{4}= & \frac{1}{1+\chi_{z z}^{(1)}}\left[-\chi_{z x}^{(1)} u_{4}-\chi_{z y}^{(1)} v_{4}+\left(B_{z x}-S_{x} B_{z z}\right) \partial_{\tau}^{2} u_{2}\right. \\
& +\left(B_{z y}-S_{y} B_{z z}\right) \partial_{\tau}^{2} v_{2}+T_{z x x}^{\prime} u_{2}^{2} \\
& \left.+\left(T_{z x y}^{\prime}+T_{z y x}^{\prime}\right) u_{2} v_{2}+T_{z y y}^{\prime} v_{2}^{2}\right]
\end{aligned}
$$

where we have set

$$
T_{z \alpha \beta}^{\prime}=T_{z \alpha \beta}-S_{\alpha} T_{z z \beta}-S_{\beta} T_{z \alpha z}+S_{\alpha} S_{\beta} T_{z z z},
$$

for $\alpha, \beta=x, y$. The definition (71) of $T_{z x x}^{\prime}$ coincides with Eq. (91) for $\alpha=\beta=x$. Then, as in the nondegenerate case, we insert $w_{4}$ into Eqs. (67) and (68) and get a system of equations for $u_{4}$ and $v_{4}$, whose homogeneous part is identical to system (38) and (39) for $u_{2}$ and $v_{2}$ and hence completely vanishes. Equations (87) and (88) can be reduced to

$$
\begin{aligned}
& 2 n c \partial_{\zeta} u_{2}-\frac{\beta_{x x}}{2} \partial_{\tau}^{3} u_{2}-\frac{\beta_{x y}}{2} \partial_{\tau}^{3} v_{2}+\Xi_{x x x} \partial_{\tau} u_{2}{ }^{2} \\
& +\Xi_{x y y} \partial_{\tau} v_{2}^{2}+2 \Xi_{x x y} \partial_{\tau} u_{2} v_{2}=0, \\
& 2 n c \partial_{\zeta} v_{2}-\frac{\beta_{y x}}{2} \partial_{\tau}^{3} u_{2}-\frac{\beta_{y y}}{2} \partial_{\tau}^{3} v_{2}+\Xi_{y x x} \partial_{\tau} u_{2}{ }^{2} \\
& +\Xi_{y y y} \partial_{\tau} v_{2}^{2}+2 \Xi_{y x y} \partial_{\tau} u_{2} v_{2}=0,
\end{aligned}
$$

which is a set of two coupled $\mathrm{KdV}$ equations. We have set

$$
\beta_{\alpha \beta}=2\left(B_{\alpha \beta}-S_{\alpha} B_{\beta z}-S_{\beta} B_{\alpha z}+S_{\alpha} S_{\beta} B_{z z}\right)
$$

and

$$
\Xi_{\alpha \beta \gamma}=\frac{1}{2}\left(\Xi_{\alpha \beta \gamma}^{\prime}+\Xi_{\alpha \gamma \beta}^{\prime}\right)
$$

where

$$
\Xi_{\alpha \beta \gamma}^{\prime}=T_{\alpha \beta \gamma}-S_{\beta} T_{\alpha z \gamma}-S_{\gamma} T_{\alpha \beta z}+S_{\beta} S_{\gamma} T_{\alpha z z}-S_{\alpha} T_{z \beta \gamma}^{\prime},
$$

for $\alpha, \beta=x, y$. Note that $\beta_{2}$ defined by Eq. (74) coincides with $\beta_{x x}$ and that $\Xi$ defined by Eq. (73) coincides with $\Xi_{x x x}$.

Using Eq. (75), we see that

$$
\beta_{\alpha \beta}=\left.\vec{d}_{\alpha} \cdot \frac{d^{2} \chi^{(1)}}{d \omega^{2}}\right|_{\omega=0} \cdot \vec{d}_{\beta},
$$

where

$$
\vec{d}_{x}=\left(\begin{array}{c}
1 \\
0 \\
-S_{x}
\end{array}\right) \quad \text { and } \quad \vec{d}_{y}=\left(\begin{array}{c}
0 \\
1 \\
-S_{y}
\end{array}\right) .
$$

As in the nondegenerate case, computing the second derivative of $\chi_{\alpha \beta}^{\prime}$ defined by Eq. (40) and taking into account the fact that the first-order derivatives of $\chi_{\alpha \beta}^{(1)}$ vanish for $\omega=0$ shows that

$$
\beta_{\alpha \beta}=\left.\frac{d^{2} \chi_{\alpha \beta}^{\prime}}{d \omega^{2}}\right|_{\omega=0}
$$

The vanishing of $\chi_{x y}^{\prime}$ may be due to the symmetry, as in the case of propagation along the optical axis of a uniaxial crystal, or perpendicular to one of the eigenaxes of a biaxial crystal. It can also depend merely on specific values of the three indices, if the coincidence of the two indices occurs accidentally in some direction which is not simply related to the eigenaxes. In the latter case, there is no reason that neither the coincidence of indices nor the vanishing of $\chi_{x y}^{\prime}$ occurs at some frequency other than the selected one (which is the limit $\omega \longrightarrow 0$ due to the long-wave approximation), and consequently the second derivatives do not coincide: $\beta_{x x} \neq \beta_{y y}$ and $\beta_{x y} \neq 0$. If, on the contrary, the coincidence of the two indices is due to symmetry, it occurs at any frequency, and the second derivatives coincide: $\beta_{x x}=\beta_{y y}$ and $\beta_{x y}=0$. An accidental coincidence of the indices in a configuration which fixes the eigenpolarizations is also possible, then $\beta_{x x} \neq \beta_{y y}$ but $\beta_{x y}=0$.

The nonlinear coefficients can be reduced by using the expression (78) of the second-order susceptibility $\chi^{(2)}$ and Eq. (79). It is seen that

$$
\Xi_{\alpha \beta \gamma}^{\prime}=\vec{d}_{\alpha} \cdot T: \vec{d}_{\beta}, \vec{d}_{\gamma}
$$

and consequently

$$
\Xi_{\alpha \beta \gamma}=\vec{d}_{\alpha} \cdot \chi^{(2)}(0 ; 0,0): \vec{d}_{\beta}, \vec{d}_{\gamma} .
$$

\section{SOLITONS}

\section{A. Linear eigenpolarizations in degenerate case}

Let us seek solutions to system (92) and (93), which represent linearly polarized waves; that is, we seek solutions of the form

$$
u_{2}=U \cos \varphi, \quad v_{2}=U \sin \varphi,
$$

in which the angle $\varphi$ determines the direction of the wave polarization. Inserting Eq. (102) into system (92) and (93), it is found that $U$ satisfies a single $\mathrm{KdV}$ equation if the two conditions

$$
\begin{gathered}
\beta_{x x}+\beta_{x y} \tan \varphi=\beta_{y y}+\beta_{x y} \operatorname{cotan} \varphi, \\
\tan ^{3} \theta \Xi_{x y y}+\tan ^{2} \theta\left(2 \Xi_{x x y}-\Xi_{y y y}\right) \\
+\tan \theta\left(\Xi_{x x x}-2 \Xi_{y x y}\right)-\Xi_{y x x}=0
\end{gathered}
$$

are satisfied. Hence in general, such an eigenpolarization of the nonlinear propagation equations do not exist, since we have two conditions for a single variable. However, if $\beta_{x y}=0$ and $\beta_{x x}=\beta_{y y}$ (i.e., if the dispersion terms do not effectively show the anisotropy), the condition (103) is automatically satisfied. Condition (104) is a third-order polynomial equation for $\tan \theta$; hence it has either 1 or 3 real solutions. This means that, in the considered conditions, either one or three eigenpolarizations of the nonlinear propagation problem exist. For such a solution, $U$ satisfies the $\mathrm{KdV}$ equation

$$
2 n c \partial_{\zeta} U-\frac{\beta}{2} \partial_{\tau}^{3} U+\Xi \partial_{\tau} U^{2}=0
$$

with $\beta=\beta_{x x}=\beta_{y y}$ and

$$
\begin{aligned}
\Xi & =\Xi_{x x x} \cos \varphi+\Xi_{x y y} \sin \varphi \tan \varphi+2 \Xi_{x x y} \sin \varphi \\
& =\Xi_{y x x} \cos \varphi \operatorname{cotan} \varphi+\Xi_{y y y} \sin \varphi+2 \Xi_{y x y} \cos \varphi .
\end{aligned}
$$

\section{B. Soliton formation from FCP input}

Rather than specifying some particular material, we prefer to discuss solution of the $\mathrm{KdV}$ equation (72) or (105) in the 
standard dimensionless form

$$
f_{Z}+6 f \partial_{T} f+\partial_{T}^{3} f=0
$$

which is obtained by means of he linear transform

$$
Z=\frac{-\beta_{2} \zeta}{4 n c T_{0}^{3}}, \quad T=\frac{\tau}{T_{0}}, \quad \varphi=\frac{-2 \Xi T_{0}^{2}}{3 \beta_{2}} u_{2},
$$

in which $T_{0}$ is some reference time. The well-known soliton of the $\mathrm{KdV}$ equation (107) is

$$
f=2 p^{2} \operatorname{sech}^{2}\left(p T-4 p^{3} Z\right)
$$

where $p$ is an arbitrary parameter. It was first derived in 1872 [59]. The KdV equation can be solved by means of the inverse scattering transform (IST) method [60], which shows that any input evolves into a finite set of solitons, plus some dispersive wave, called 'radiation' in the terminology of the mathematical theory of solitons. We have solved numerically the $\mathrm{KdV}$ equation (107) by means of the modified Euler exponential time differencing scheme [61], starting from an initial data in the form of a FCP, as

$$
f=\mathcal{A} \cos (\omega T+\psi) \operatorname{sech}^{2}\left(T / T_{0}\right) .
$$

We take as a reference time $T_{0}=0.8 \mathrm{fs}$, so that a dimensionless angular frequency $\omega=1.5$ corresponds to a wavelength very close to $1 \mu \mathrm{m}$. In the most simple geometry, $\Xi$ coincides with $\chi_{\alpha \alpha \alpha}^{(2)}, \alpha$ being the polarization direction. We can take as a typical value of $\Xi$ the value of $\chi_{y y y}^{(2)}$ in beta barium borate (BBO) [i.e., 4.4 pm/V (cf. [58], p. 50)]. Sellmeier approximations of the dispersion relation for $\mathrm{BBO}$ are given by industrials selling such crystals (we use the data given by Red Optronics [62]). Straightforward calculations give then the value of $\beta_{2}$, we get $\beta_{2}=-0.0111$ and $-0.0180 \mathrm{fs}^{2}$ for the ordinary and extraordinary indices, respectively, at $\lambda=1 \mu \mathrm{m}$. We retain the largest of these two values. It must be noted that we do not claim that $\mathrm{BBO}$ is the best candidate for the experimental realization of the present theory. Other crystals commonly used for second-harmonic generation, such as potassium dihydrogen phosphate (KDP) or silver gallium sulfide $\left(\mathrm{AgGaS}_{2}\right)$ may be considered in the same way; the key questions of the applicability of the theory are that the transparency domain of the medium is sufficient, and that it actually supports the huge instantaneous intensity of the FCP. Many secondary questions arise, such as the actual description of the dispersion, since the present model does not take into account the resonances at large wavelengths, due to the a longwave approximation. Only real experiments can answer the question of to what extent the theory should be corrected on this point. We use the values pertaining to BBO as typical orders of magnitude. We consider normalized amplitudes as 3.5 and 5, which corresponds according to Eq. (108) to maximum electric field $E_{m}=3.4$ and $4.8 \times 10^{10} \mathrm{~V} / \mathrm{m}$, respectively. Using $I_{m}=c \epsilon_{0} E_{m}^{2}$ as the expression of the instantaneous intensity, it corresponds to $I_{m}=3$ and $6 \times 10^{14} \mathrm{~W} / \mathrm{cm}^{2}$, which belongs to the range of huge values of intensities that can be reached in the few-cycle regime.

As expected from the IST theory, the input FCP evolves into a few solitons, plus a dispersive wave. The number of solitons depend on the amplitude and duration of the initial pulse and on the carrier envelope phase. Two typical examples are shown in Figs. 1 and 2. Although an appreciable part of the energy is dispersed, high-amplitude solitons are formed. The influence of the carrier-envelope phase $\psi$ is illustrated on Fig. 1: whereas for a cosine-type pulse (i.e., $\psi=0$ ) or for $\psi$ close to zero the typical outcome is formed by a single soliton or, more generally, one soliton much larger than all others, two identical solitons are formed for $\psi=\pi$. It it seen in Fig. 1(b) that the two solitons have the same velocity. For the most narrow and energetic pulses of the cosine type, as we said, a single soliton is formed, or several solitons among which one strongly dominates. If the FCP duration is a bit larger and if its amplitude is not too large, the difference between the leading soliton and the smaller ones may be less important, as seen in Fig. 2, which shows the formation of three solitons: a larger one and two smaller ones.

A quite important part of the energy is converted into dispersive waves. In the $(T, Z)$ frame, the solitons propagate forward and the dispersive waves backward. Recall that the variable $T$ is the retarded time (i.e., the figures are plotted in

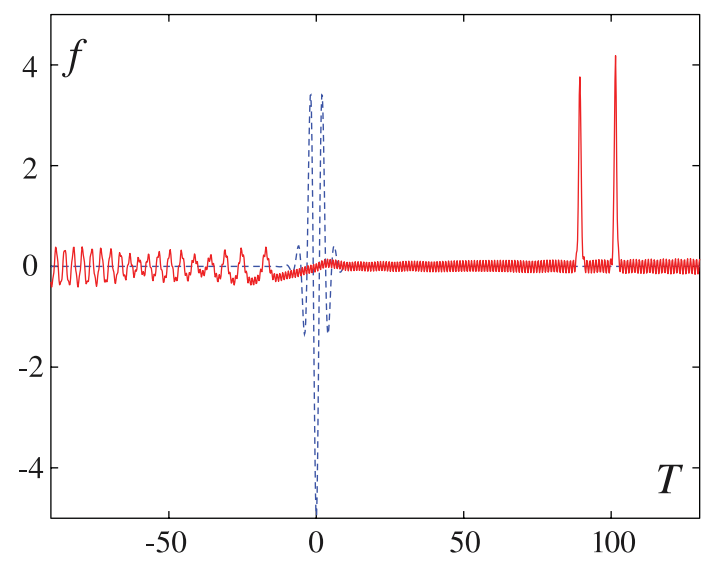

(a)

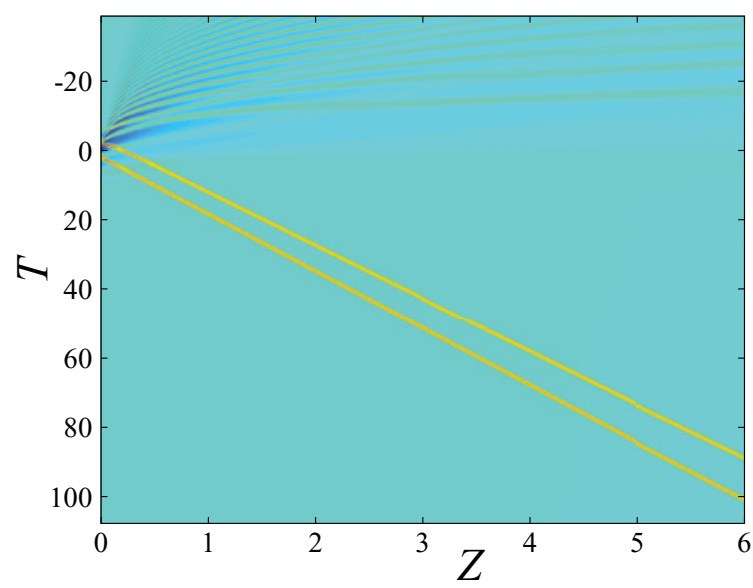

(b)

FIG. 1. (Color online) Formation of two solitons from a FCP input. Panel (a) shows the wave profile. Dashed line is input $(Z=0$ ), solid line is output ( $Z=6$ ). Panel (b) shows the evolution. The dimensionless parameters are amplitude $\mathcal{A}=5$, angular frequency $\omega=1.5$, carrier-envelope phase $\psi=\pi$, duration $T_{0}=3.2$ (dimensionless). 


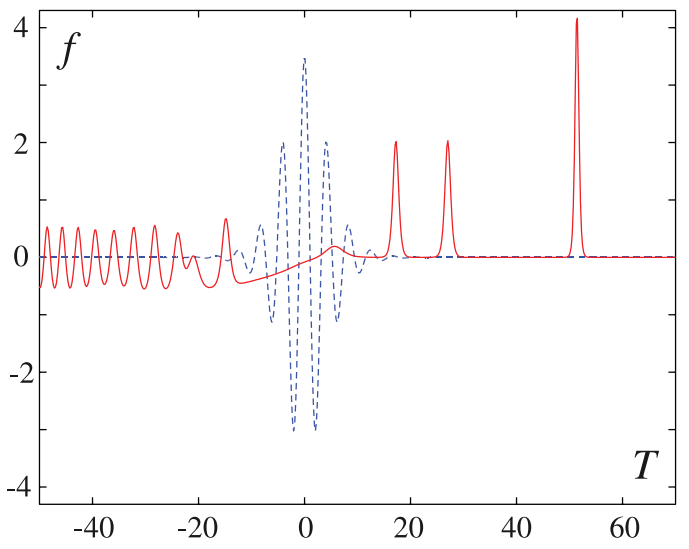

(a)

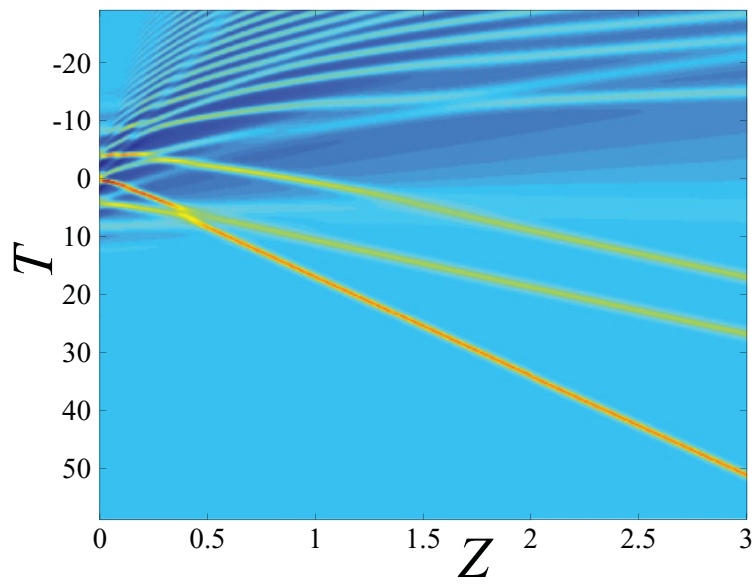

(b)

FIG. 2. (Color online) Formation of three solitons from a FCP input. Panel (a) shows the wave profile. Dashed line is input $(Z=0$ ), solid line is output ( $Z=3$ ). Panel (b) shows the evolution. The dimensionless parameters are amplitude $\mathcal{A}=3.5$, angular frequency $\omega=1.5$, carrier-envelope phase $\psi=0$, duration $T_{0}=5.33$ (dimensionless).

the frame moving at the velocity $V$ ). It is defined as $V=c / n$, where the index $n$ is given by Eq. (43). Exactly, $V$ is both the linear group and phase velocities at the limit of large wavelengths. If $\beta_{2}$ is negative, $Z$ and $\zeta$ are in same direction: the solitons propagate slower than $V$, the dispersive waves faster. If, on the contrary, $\beta_{2}$ is positive, $Z$ and $\zeta$ are in opposite directions and the results are inverted: the solitons propagate faster than $V$. However, backward-propagating waves can also be formed, which cannot accounted for by the KdV model, which is unidirectional. Especially, when a FCP coming from vacuum is launched into a $\chi^{(2)}$ medium, reflection occurs at the interface, and nonlinear effects may increase the reflected part $[47,49]$. Nonlinear backward diffusion distributed in the medium may also arise, but it remains very small with respect to refection at the interface. Although in some situations forward and backward nonlinear diffusions may compensate, leading to the formation of a lossless traveling wave [35], backward diffusion may be understood as one of the absorption and diffusion mechanisms responsible for the damping of the wave, which was neglected in the present approach.

\section{CONCLUSIONS}

In conclusion, in this work we have studied in detail the propagation of ultrashort pulses in noncentrosymmetric nonlinear optical media described by a general Hamiltonian of multilevel atoms. Assuming that all transition frequencies of the medium are well above the typical wave frequency, we used a long-wave approximation to derive a governing model describing ultrashort soliton propagation in a quadratic nonlinear medium.

The result depends on the difference between the two indices of the medium in the considered propagation direction. If this difference is large enough, so that the two eigenpolarizations cannot interact, a generic Korteweg-de Vries model is derived. It describes the decay of a FCP input into one or a few unipolar half-cycle solitons. If the difference between the two indices can be neglected, a generic coupled system of Korteweg-de Vries equations describing ultrashort soliton evolution in quadratic materials was derived by using a rigorous application of the multiscale analysis.

However, in a real nonlinear optical medium, a broad transparency range is required, and hence all atomic transitions must be far enough from the pulse central frequency. However, in such materials not all transitions belong to the ultraviolet domain. The contribution of the infrared transitions, yielding a sine-Gordon model in the case of two-level atoms and cubic nonlinearity, which are not taken into account in the present work, should be considered in a further study by means of a short-wave approximation applied to the same general quantum mechanical model.

\section{ACKNOWLEDGMENTS}

The work of D.M. was supported in part by a Senior Chair Grant from the Région Pays de Loire, France. Support from the Romanian Ministry of Education and Research (Project PN-II-ID-PCE-2011-3-0083) is also acknowledged.
[1] L. Gallmann, D. H. Sutter, N. Matuschek, G. Steinmeyer, U. Keller, C. Iaconis, and I. A. Walmsley, Opt. Lett. 24, 1314 (1999); U. Morgner, F. X. Kärtner, S. H. Cho, Y. Chen, H. A. Haus, J. G. Fujimoto, E. P. Ippen, V. Scheuer, G. Angelow, and T. Tschudi, ibid. 24, 411 (1999); D. H. Sutter, G. Steinmeyer,
L. Gallmann, N. Matuschek, F. Morier-Genoud, U. Keller, V. Scheuer, G. Angelow, and T. Tschudi, ibid. 24, 631 (1999); A. Shirakawa, I. Sakane, M. Takasaka, and T. Kobayashi, App. Phys. Lett. 74, 2268 (1999).

[2] T. Brabek and F. Krausz, Rev. Mod. Phys. 72, 545 (2000). 
[3] E. Goulielmakis, M. Schultze, M. Hofstetter, V. S. Yakovlev, J. Gagnon, M. Uiberacker, A. L. Aquila, E. M. Gullikson, D. T. Attwood, R. Kienberger, F. Krausz, and U. Kleineberg, Science 320, 1614 (2008).

[4] M. Wegener, Extreme Nonlinear Optics (Springer, Berlin, 2005).

[5] A. Scrinzi, M. Yu. Ivanov, R. Kienberger, and D. M. Villeneuve, J. Phys. B 39, R1 (2006).

[6] F. Krausz and M. Ivanov, Rev. Mod. Phys. 81, 163 (2009).

[7] M. Drescher, M. Hentschel, R. Kienberger, G. Tempea, C. Spielmann, G. A. Reider, P. B. Corkum, and F. Krausz, Science 291, 1923 (2001); M. Hentschel, R. Kienberger, C. Spielmann, G. A. Reider, N. Milosevic, T. Brabec, P. Corkum, U. Heinzmann, M. Drescher, and F. Krausz, Nature (London) 414, 509 (2001); P. M. Paul, E. S. Toma, P. Breger, G. Mullot, F. Augé, P. Balcou, H. G. Muller, and P. Agostini, Science 292, 1689 (2001).

[8] R. Kienberger, Zenghu Chang, and Chang Hee Nam, J. Phys. B 45, 070201 (2012).

[9] N. N. Rosanov, V. E. Semenov, and N. V. Vyssotina, Laser Phys. 17, 1311 (2007).

[10] N. N. Rosanov, V. E. Semenov, and N. V. Vysotina, Quantum Electron. 38, 137 (2008).

[11] A. Nazarkin, Phys. Rev. Lett. 97, 163904 (2006).

[12] A. I. Maimistov, Quantum Electron. 40, 756 (2010).

[13] T. Brabec and F. Krausz, Phys. Rev. Lett. 78, 3282 (1997).

[14] M. V. Tognetti and H. M. Crespo, J. Opt. Soc. Am. B 24, 1410 (2007).

[15] A. A. Voronin and A. M. Zheltikov, Phys. Rev. A 78, 063834 (2008).

[16] P. Kinsler and G. H. C. New, Phys. Rev. A 67, 023813 (2003).

[17] A. A. Zozulya, S. A. Diddams, and T. S. Clement, Phys. Rev. A 58, 3303 (1998).

[18] N. Aközbek, M. Scalora, C. M. Bowden, and S. L. Chin, Opt. Commun. 191, 353 (2001).

[19] J. E. Rothenberg, Opt. Lett. 17, 1340 (1992).

[20] J. K. Ranka and A. L. Gaeta, Opt. Lett. 23, 534 (1998).

[21] E. M. Belenov and A. V. Nazarkin, JETP Lett. 51, 288 (1990) [Pis'ma Zh. Eksp. Teor. Fiz. 51, 252 (1990)].

[22] A. I. Maimistov and S. O. Elytin, J. Modern Opt. 39, 2201 (1992).

[23] A. E. Kaplan and P. L. Shkolnikov, Phys. Rev. Lett. 75, 2316 (1995).

[24] M. A. Porras, Phys. Rev. A 60, 5069 (1999).

[25] S. V. Sazonov, JETP 92, 361 (2001).

[26] A. I. Maimistov, Opt. Spektrosk. 76, 636 (1994) [Opt. Spectrosc. 76, 569 (1994)].

[27] I. V. Mel'nikov, D. Mihalache, F. Moldoveanu, and N. C. Panoiu, Phys. Rev. A 56, 1569 (1997); JETP Lett. 65, 393 (1997).

[28] I. V. Mel'nikov, D. Mihalache, and N. C. Panoiu, Opt. Commun. 181, 345 (2000).

[29] H. Leblond and F. Sanchez, Phys. Rev. A 67, 013804 (2003).

[30] I. V. Mel'nikov, H. Leblond, F. Sanchez, and D. Mihalache, IEEE J. Sel. Topics Quantum Electron. 10, 870 (2004); H. Leblond, F. Sanchez, I. V. Mel'nikov, and D. Mihalache, Math. Comp. in Simulation 69, 378 (2005).

[31] H. Leblond, S. V. Sazonov, I. V. Mel'nikov, D. Mihalache, and F. Sanchez, Phys. Rev. A 74, 063815 (2006).
[32] H. Leblond, I. V. Mel'nikov, and D. Mihalache, Phys. Rev. A 78, 043802 (2008).

[33] H. Leblond and D. Mihalache, Phys. Rev. A 79, 063835 (2009); J. Optoelectron. Adv. Mat. 12, 1 (2010); Rom. Rep. Phys. 63, 1254 (2011).

[34] S. A. Skobelev, D. V. Kartashov, and A. V. Kim, Phys. Rev. Lett. 99, 203902 (2007).

[35] Sh. Amiranashvili, A. G. Vladimirov, and U. Bandelow, Phys. Rev. A 77, 063821 (2008).

[36] R. K. Dodd, J. C. Eilbeck, J. D. Gibbon, and H. C. Morris, Solitons and Nonlinear Wave Equations (Academic, London, 1982).

[37] M. J. Ablowitz and H. Segur, Solitons and the Inverse Scattering Transform (SIAM, Philadelphia, 1981).

[38] K. Konno, W. Kameyama, and H. Sanuki, J. Phys. Soc. Jpn. 37, 171 (1974).

[39] A. M. Kosevich and A. S. Kovalev, Solid State Commun. 12, 763 (1973).

[40] E. V. Kazantseva and A. I. Maimistov, Phys. Lett. A 263, 434 (1999).

[41] E. V. Kazantseva, A. I. Maimistov, and B. A. Malomed, Opt. Commun. 188, 195 (2001).

[42] H. Leblond, Phys. Rev. A 78, 013807 (2008).

[43] L. Bergé and S. Skupin, Phys. Rev. Lett. 100, 113902 (2008).

[44] H. Leblond, D. Kremer, and D. Mihalache, Phys. Rev. A 80, 053812 (2009).

[45] H. Leblond, D. Kremer, and D. Mihalache, Phys. Rev. A 81, 033824 (2010).

[46] H. Leblond and D. Mihalache, Phys. Rev. A 81, 063815 (2010); D. Mihalache, Rom. J. Phys. 57, 352 (2012).

[47] N. N. Rosanov, V. V. Kozlov, and S. Wabnitz, Phys. Rev. A 81, 043815 (2010)

[48] H. Leblond and D. Mihalache, J. Phys. A: Math. Theor. 43, 375205 (2010).

[49] V. V. Kozlov, N. N. Rosanov, C. De Angelis, and S. Wabnitz, Phys. Rev. A 84, 023818 (2011).

[50] A. Pusch, J. M. Hamm, and O. Hess, Phys. Rev. A 84, 023827 (2011).

[51] E. D. Farnum and J. N. Kutz, Opt. Lett. 35, 3033 (2010).

[52] A. V. Kim and S. A. Skobelev, Phys. Rev. A 83, 063832 (2011).

[53] H. Leblond, H. Triki, F. Sanchez, and D. Mihalache, Phys. Rev. A 83, 063802 (2011).

[54] H. Leblond, H. Triki, F. Sanchez, and D. Mihalache, Opt. Commun. 285, 356 (2012).

[55] H. Leblond, H. Triki, and D. Mihalache, Phys. Rev. A 84, 023833 (2011).

[56] H. Triki, H. Leblond, and D. Mihalache, Opt. Commun. 285, 3179 (2012).

[57] H. Leblond, J. Phys. B 41, 043001 (2008).

[58] R. W. Boyd, Nonlinear Optics, 3rd ed. (Academic Press, San Diego, 2007).

[59] J. Boussinesq, Journal de Mathématiques Pures et Appliqués Sér. II 17, 55 (1872).

[60] C. S. Gardner, J. M. Greene, M. D. Kruskal, and R. M. Miura, Phys. Rev. Lett. 19, 1095 (1967).

[61] S. M. Cox and P. C. Matthews, J. Comput. Phys. 176, 430 (2002).

[62] [http://www.redoptronics.com/BBO-crystal.html]. 